\title{
Nicht mit Wolle oder Plastik ins Bett!
}

\author{
Wie man Babys bettet, so sensibilisiert man sie. Diese These über- \\ prüften australische Epidemiologen, indem sie mit großem \\ statistischen Aufwand den Einfluss von Schlafunterlagen bei \\ Säuglingen auf die Sensibilisierung gegen Hausstaubmilben im \\ Kindesalter untersuchten.
}

E in weiches Schaffell bietet einem Baby Wärme und Geborgenheit. Doch könnte man meinen, dass Schaffelle eher zu einer Sensibilisierung füh-

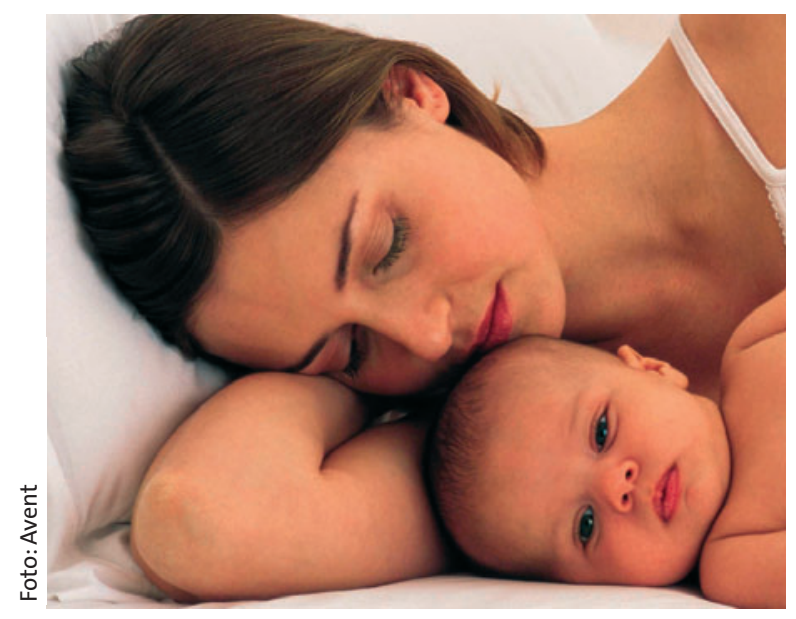

Was ist allergologisch fragwürdiger, ein Baby auf ein Schaffell zu betten oder eine Plastikunterlage im Bett zu verwenden? Oder ist eine private Krankenversicherung gar das größere Risiko für eine Sensibilisierung? ren als Plastikunterlagen, da sie mehr Milben enthalten. Falsch vermutet: Beide Unterlagen erhöhen das relative Risiko einer Sensibilisierung gegen Hausstaubmilben etwa um den Faktor 2 im Vergleich zu sonstigen Schlafunterlagen.

Um zu diesem Ergebnis zu gelangen, wurden die Eltern von insgesamt 498 Babys kurz nach der Geburt zu einer großen Anzahl von Kriterien befragt, zu denen neben Angaben über Schlafunterlagen auch solche über das Rauchverhalten, über die Anzahl der Geschwister, über die Familienanamnese, aber auch über die Art der Krankenversicherung der Eltern und noch vieles an- dere mehr gehörten. Als die Kinder 8 Jahre alt waren, überprüften die Epidemiologen dann die Sensibilisierung gegen Hausstaubmilben mittels Pricktest: $31,5 \%$ der Kinder reagierten positiv.

Während viele andere Faktoren keinen signifikanten Einfluss hatten, war das Risiko sowohl bei Schaffellen als auch bei Plastikunterlagen, aber auch bei einer privaten Krankenversicherung signifikant erhöht. Diese relative Risikoerhöhung blieb auch nach Adjustierung für diverse andere Kriterien bestehen.

Die Autoren diskutieren den unerwarteten Befund vor dem Hintergrund möglicher schädlicher Ausdünstungen des Plastiks, die den Effekt der Allergenreduktion ohne Schaffell durch eine Lungenreizung wieder kompensieren könnten. Das Ergebnis der Privatversicherung als Risikofaktor diskutieren die Autoren nicht.

Fazit: Sowohl Schaffelle als auch Plastikunterlagen erhöhen das Risiko einer Sensibilisierung gegen Hausstaubmilben.

cjh

Trevillian L et al. An association between plastic mattress covers and sheepskin underbedding use in infancy and house dust mite sensitization in childhood: a prospective study. Clin Exp Allergy 2003; 33: 483-9

\section{Statistiker kaufen leicht die Katze im Sack}

\section{Ob der frühkindliche Kontakt mit Katzen und Hunden vermehrt zu Allergie führt oder umgekehrt sogar vor einer Sensibilisierung schützt, ist weiterhin umstritten. Beruhen die unterschiedlichen Ergebnisse verschiedener Studien auf einer Verzerrung der Daten?}

S chwedische Forscher, u. a. vom Karolinska-Institut, befragten prospektiv die Eltern von 4.089 Kindern 2 Monate nach der Geburt des Kindes sowie am Ende des 1. und des 2. Lebensjahres zu Haustieren und Allergien in der Familie. Eindeutiges Ergebnis der Studie: Die Katze lebte viel seltener in Haushalten, in denen mindestens ein Elternteil unter Asthma oder Heuschnupfen litt (im Schnitt 4,6\%), als bei Eltern ohne allergische Erkrankungen $(11,3 \%)$. Noch seltener traten Katzen in Familien auf, in denen ein älteres Geschwister allergisch war (3,7\%). Auf die Häufigkeit von Hunden im Haushalt hatten Allergien bei Eltern und Geschwistern allerdings weniger Einfluss. Nur wenn Geschwister oder beide Eltern unter atopischem Ekzem litten, fiel die Hundehaltung von sonst 5,9\% auf $1,7 \%$ bis $1,5 \%$. Schließlich erstaunt es dann auch nicht mehr, dass die Anzahl von Haushalten mit Katze bei familiärer Belastung mit der Zeit deutlich abnimmt.

Fazit: Katzen leben eher in Haushalten, in denen keine atopischen Erkrankungen vorkommen. Untersuchungen über den Einfluss von Haustieren auf die Allergieentwicklung sollten diesen Faktor auf jeden Fall statistisch berücksichtigen, sonst könnte der Eindruck entstehen, Haustiere schützen vor Allergie.

cjh

Almqvist C et al. Hereditiy, pet ownership, and confounding control in a population-based birth cohort. J Allergy Clin Immunol 2003, 111: 800-6 